\title{
Affective Intelligence: The Human Face of AI
}

\author{
Lori Malatesta, Kostas Karpouzis, and Amaryllis Raouzaiou \\ National Technical University of Athens 15780, Zographou, Athens, Greece \\ \{kkarpou, lori, araouz\}@image.ntua.gr
}

\begin{abstract}
Affective computing has been an extremely active research and development area for some years now, with some of the early results already starting to be integrated in human-computer interaction systems. Driven mainly by research initiatives in Europe, USA and Japan and accelerated by the abundance of processing power and low-cost, unintrusive sensors like cameras and microphones, affective computing functions in an interdisciplinary fashion, sharing concepts from diverse fields, such as signal processing and computer vision, psychology and behavioral sciences, human-computer interaction and design, machine learning, and so on. In order to form relations between low-level input signals and features to high-level concepts such as emotions or moods, one needs to take into account the multitude of psychology and representation theories and research findings related to them and deploy machine learning techniques to actually form computational models of those. This chapter elaborates on the concepts related to affective computing, how these can be connected to measurable features via representation models and how they can be integrated into humancentric applications.
\end{abstract}

\section{$1 \quad$ Introduction}

As research has revealed the deep role that emotion and emotional expression play in human social interaction, researchers in human computer interaction have proposed that more effective human computer interfaces can be realized if the interface models the user's emotion as well as expresses emotions. Affective computing is computing that relates to, arises from, or deliberately influences emotion or other affective phenomena. According to Rosalind Picard's pioneering article [32], if we want computers to be genuinely intelligent and to interact naturally with us, we must give computers the ability to recognize, understand, and even to have and express emotions. These positions have become the foundations of research in the area and have been investigated in great depth after their first postulation.

Emotion is fundamental to human experience, influencing cognition, perception, and everyday tasks such as learning, communication, and even rational decision-making. Affective computing aspires to bridge the gap that typical human 
computer interaction largely ignored thus creating an often frustrating experience for people, in part because affect had been overlooked or was hard to measure.

In order to take these ideas a step further, towards the objectives of practical applications, we need to adapt methods of modelling affect to the requirements of the project's showcases. To do so it is fundamental to review prevalent psychology theories on emotion, to disambiguate their terminology and identify the fitting computational models that can allow for affective interactions in the desired environments.

\section{Terminology Disambiguation}

We speak of disambiguation since a lot of confusion exists regarding emotion research terminology, and not without a reason. Different definitions of the role and nature of emotions arise from different scientific approaches since emotion research is typically multidisciplinary. Different disciplines (i.e. psychology, cognitive neuroscience etc) provide theories and corresponding models that are based on diverse underlying assumptions, are based on different levels of abstraction and may even have different research goals altogether.

So what are emotions? It largely remains an open question. Some define it as the physiological changes caused in our body, while others treat it as a purely intellectual thought-process.

In psychology research [36] the term 'affect' is very broad, and has been used to cover a wide variety of experiences such as emotions, moods, and preferences. In contrast, the term 'emotion' [9] tends to be used to refer to fairly brief but intense experiences although it is also used in a broader sense. Finally, moods or states describe low-intensity but more prolonged experiences.

From a cognitive neuroscience point of view, Damasio [10] makes a distinction between emotions, which are publicly observable body states, and feelings, which are mental events observable only to the person having them. Based on neuroscience research he and others have done, Damasio argues that an episode of emotion begins with an emotionally "competent" stimulus (such as an attractive person or a scary house) that the organism automatically appraises as conducive to survival or well-being (a good thing) or not conducive (bad). This appraisal takes the form of a complex array of physiological reactions (e.g., quickening heartbeat, tensing facial muscles), which is mapped in the brain. From that map, a feeling arises as "an idea of the body when it is perturbed by the emoting process" [10].

It is apparent that there is no right or wrong approach, and an attempt at a full terminology disambiguation would not be possible without biasing our choices towards one theory over the other. This is to make the point that the context of each approach has to be carefully defined. Next we are going to enumerate core elements of emotion and ways to distinguish them from other affective phenomena. This will lead us to a short description of the directions of affective computing. Subsequently we will put forward the most prevalent psychological theories of 
emotion along with corresponding computational modelling approaches and couple them to the affective computing goals and more specifically to the goals of practical applications.

\subsection{Defining 'Emotion' and 'Feeling'}

Emotion, according to Klaus Scherer ([40], [42]), can be defined as an episode of interrelated, synchronized changes in the states of all or most of five organismic subsystems in response to the evaluation of an external or internal stimulus event as relevant to major concerns of the organism. The components of an emotion episode are the particular states of the subsystems mentioned. The process consists of the coordinated changes over time.

Most current psychological theories postulate that subjective experience, peripheral physiological response patterns, and motor expression are major components of emotion. These three components have often been called the emotional response triad. Some theorists include the cognitive and motivational domains as components of the emotion process. The elicitation of action tendencies and the preparation of action have also been implicitly associated with emotional arousal. However, only after explicit inclusion of motivational consequences in theories (and Frijda's forceful claim for the emotion-differentiating function of action tendencies, see [14]), have these important features of emotion acquired the status of a major component. The inclusion of a cognitive information-processing component has met with less consensus. Many theorists still prefer to see emotion and cognition as two independent but interacting systems. However, one can argue that all subsystems underlying emotion components function independently much of the time, and that the special nature of emotion as a hypothetical construct consists of the coordination and synchronization of all these systems during an emotion episode [43].

How can emotions, as defined above, be distinguished from other affective phenomena such as feelings, moods, or attitudes? Let us take the term feeling first. Scherer aligns feeling with the "subjective emotional experience" component of emotion, thus reflecting the total pattern of cognitive appraisal as well as motivational and somatic response patterning that underlie the subjective experience of an emotion. If we use the term feeling, a single component denoting subjective experience process, as a synonym for emotion (the total multi-modal component process), this is likely to produce serious confusion and hamper our understanding of the phenomenon.

If we accept feeling as one of emotion's components, then the next step is to differentiate emotion from other types of affective phenomena. Instances of these phenomena, which can vary in degree of affectivity, are often called "emotions" in the literature. According to Scherer [44], there are five such types of affective phenomena that should be distinguished from emotion: preferences, attitudes, moods, affective dispositions and interpersonal stances.

In order to differentiate emotions from the rest of the affective phenomena we shall sketch out core elements of emotions. 


\subsubsection{Event Focus}

Emotions are generally elicited by stimulus events. Something happens to the organism, which, after having been evaluated for its significance, stimulates or triggers a response. Often such events will consist of natural phenomena like thunderstorms, or the behaviour of other people or animals that may have significance for our well-being. In other cases, one's own behaviour can be the event that elicits emotion, as in the case of pride, guilt, or shame. In addition to such events that are more or less external to the organism, internal events are explicitly considered as emotion elicitors. These may consist of sudden neuro-endocrine or physiological changes or, more typically, of memories or images that might come to our mind. These recalled or imagined representations of events can be sufficient to generate strong emotions [18]. The event focus element means that emotions need to be somehow connected or anchored to a specific event, external or internal, rather than being free-floating, resulting from a strategic or intentional decision, or existing as a permanent characteristic of an individual.

\subsubsection{Appraisal Basis}

A central aspect of the component process definition of emotion is that the eliciting event and its consequences must be relevant to major concerns of the individual. This seems rather obvious; as we do not generally get emotional about things or people we do not care about. Frijda [14] talks of emotions as relevance detectors. Componential theories of emotion generally assume that the relevance of an event is determined by a rather complex, yet very rapidly occurring evaluation process that can take place on several levels of processing, ranging from automatic and implicit to conscious conceptual or propositional evaluations [27]. It makes sense to distinguish between intrinsic and extrinsic appraisal. Intrinsic appraisal evaluates the features of an object or person independently of the current needs and goals of the appraiser, based on genetic preferences (e.g. sweet taste) or learned preferences (e.g., bittersweet food) (see [40], [41]). Extrinsic appraisal (also known as transactional appraisal; see [26]) evaluates events and their consequences in terms of their contribution to the salient needs, desires, or goals of the appraiser.

\subsubsection{Response Synchronization}

This element is also implied by the adaptive functions of emotion. If emotions prepare appropriate responses to events, the response patterns must correspond to the appraisal analysis of the presumed implications of the event. Given the importance of the eliciting event, which disrupts the flow of behaviour, all or most of the subsystems of the organism must contribute to response preparation. The resulting massive mobilization of resources must be coordinated, a process which can be described as response synchronization [42]. This is in fact one of the most important design features of emotion, one that in principle can be operationalized and measured empirically. 


\subsubsection{Rapidity of Change}

Events, and particularly their appraisal, change rapidly, often because of new information, or due to re-evaluation. As appraisal drives the patterning of the responses in the interest of adaptation, the emotional response patterning is also likely to change rapidly. While we are in the habit of talking about "emotional states", these are rarely steady states. Rather, emotion processes are undergoing constant modification, allowing rapid readjustment to changing circumstances or evaluations.

\subsubsection{Behavioural Impact}

Emotions prepare the ground for adaptive action tendencies and their motivational underpinnings. In this sense, they have a strong effect on behaviour resulting from emotion. They often interrupt ongoing behaviour sequences, and generate new goals and plans. In addition, the motor expression component of emotion has a strong impact on communication. This may have important consequences for social interaction.

\subsubsection{Intensity}

Given the importance of emotions for behavioural adaptation, one can assume the intensity of the response patterns and the corresponding emotional experience to be relatively high. This may be an important design feature in distinguishing emotions from moods, for example.

\subsubsection{Duration}

As emotions imply massive response mobilization and synchronization as part of specific action tendencies, their duration must be relatively short in order not to tax the resources of the organism, and to allow behavioural flexibility. In contrast, low-intensity moods that have little impact on behaviour can be maintained for much longer periods without there being adverse effects.

\section{Distinguishing 'Emotion' from Other Affective Phenomena}

\subsection{Features of Other Affective Phenomena}

Having presented the basic elements of emotions it is now possible to define the other phenomena mentioned earlier in such a way as to distinguish them from emotions. 


\subsubsection{Preferences}

We will refer to relatively stable evaluative judgments in the sense of liking or disliking a stimulus, or preferring it to other objects or stimuli, as preferences. By definition, stable preferences should generate intrinsic appraisal independently of current needs or goals, although the latter might modulate the appraisal [41]. The affective states produced by encountering attractive or aversive stimuli (event focus) are stable, of relatively low intensity, and do not produce pronounced response synchronization. Preferences generate unspecific positive or negative feelings, with low behavioural impact, except for tendencies towards approach or avoidance.

\subsubsection{Attitudes}

Relatively enduring beliefs and predispositions towards specific objects or persons are generally called attitudes. Social psychologists have long identified three components of attitudes (see [4]): a) a cognitive component (beliefs about the attitude object), b) an affective component (consisting mostly of differential valence), and c) a motivational or behavioural component (a stable action tendency with respect to the object, e.g., approach or avoidance). Attitude objects can be things, events, persons, and groups or categories of individuals. Attitudes do not need to be triggered by event appraisals, although they may become more salient when encountering or thinking of the attitude object. The affective states induced by a salient attitude can be labelled by terms such as "hating", "valuing", or "desiring". Intensity and response synchronization are generally weak, and behavioural tendencies are often overridden by situational constraints.

\subsubsection{Moods}

Emotion psychologists have often discussed the difference between mood and emotion (e.g. [15]). Generally, moods are considered as diffuse affect states, characterized by a relatively enduring predominance of certain types of subjective feelings that affect the experience and behaviour of a person. Moods may often develop without an apparent cause that could be clearly linked to an event or specific appraisal. They are generally of low intensity and show little response synchronization, but may last for hours or even days. Examples are being cheerful, gloomy, listless, depressed, or buoyant.

\subsubsection{Affective Dispositions}

Many stable personality traits and behaviour tendencies have a strong affective core (e.g., being nervous, anxious, irritable, reckless, morose, hostile, envious or jealous). These dispositions describe the tendency of a person to experience cer- 
tain moods more frequently or to be prone to react with certain types of emotions, even upon slight provocation. Not surprisingly, terms like "irritable" or "anxious" can describe both affect dispositions and momentary moods or emotions. It is important to specify whether the term is used to qualify personality disposition or an episodic state. Affect dispositions also include emotional pathology: while being in a depressed mood is quite normal, being constantly depressed may be a sign of an affective disturbance requiring medical attention.

\subsubsection{Interpersonal Stances}

This category refers to an affective style that spontaneously develops or is strategically employed in the interaction with a person or a group of persons, colouring the interpersonal exchange in that situation (e.g. being polite, distant, cold, warm, supportive, contemptuous). Interpersonal stances are often triggered by events, such as encountering a certain person. However, they are less shaped by spontaneous appraisal than by affect dispositions, interpersonal attitudes, and, most importantly, strategic intentions. Thus, when an irritable person encounters a disliked individual, that person is more likely to adopt an interpersonal stance of hostility in the interaction, as compared to an agreeable person.

\subsection{Emotions in Applied Intelligence}

Having distinguished emotions from other types of affective phenomena it is now of particular interest, in regard to the new media domain, to present a suggested distinction on a different level. Scherer [43] questioned the need to distinguish between two different types of emotion: (1) aesthetic emotions (2) utilitarian emotions. The latter correspond to the "garden variety" of emotions usually studied in emotion research, such as anger, fear, joy, disgust, sadness, shame, guilt. These types of emotions can be considered utilitarian in the sense of facilitating our adaptation to events that have important consequences for our well-being. Such adaptive functions are the preparation of action tendencies (fight, flight), recovery and reorientation (grief, work), motivational enhancement (joy, pride), or the creation of social obligations (reparation). Because of their importance for survival and well-being, many utilitarian emotions are high-intensity emergency reactions, involving the synchronization of many subsystems, as described earlier. In the case of aesthetic emotions, adaptation to an event that requires the appraisal of goal relevance and coping potential is absent, or much less pronounced. Kant defined aesthetic experience as "disinterested pleasure" ([24]), highlighting the complete absence of utilitarian considerations. Thus, my aesthetic experience of a work of art or a piece of music is not shaped by the appraisal of the work's ability to satisfy my bodily needs, further my current goals or plans, or correspond to my social values. Rather, aesthetic emotions are produced by the appreciation of the intrinsic qualities of a work of art or an artistic performance, or the beauty of na- 
ture. Examples of such aesthetic emotions are: being moved or awed, full of wonder, admiration, bliss, ecstasy, fascination, harmony, rapture, solemnity.

This differentiation of emotions has an impact on the way an appraisal based modelling approach would be implemented. It would not make sense to try and model all the proposed components of an appraisal process in cases where only aesthetic emotions are expected. On the other hand, it would make sense to provide a deeper model in cases where anger or frustration are common emotional states such as in the example of interactive Television.

\section{$4 \quad$ Areas of Affective Computing}

Affective computing deals with the design of systems and devices which can recognize, interpret, and process emotions. We are going to fledge out the potentials this research domain can provide in the field of new media applications and identify the matching theoretical background that will act as a tool for effectively modelling emotional interaction in such environments.

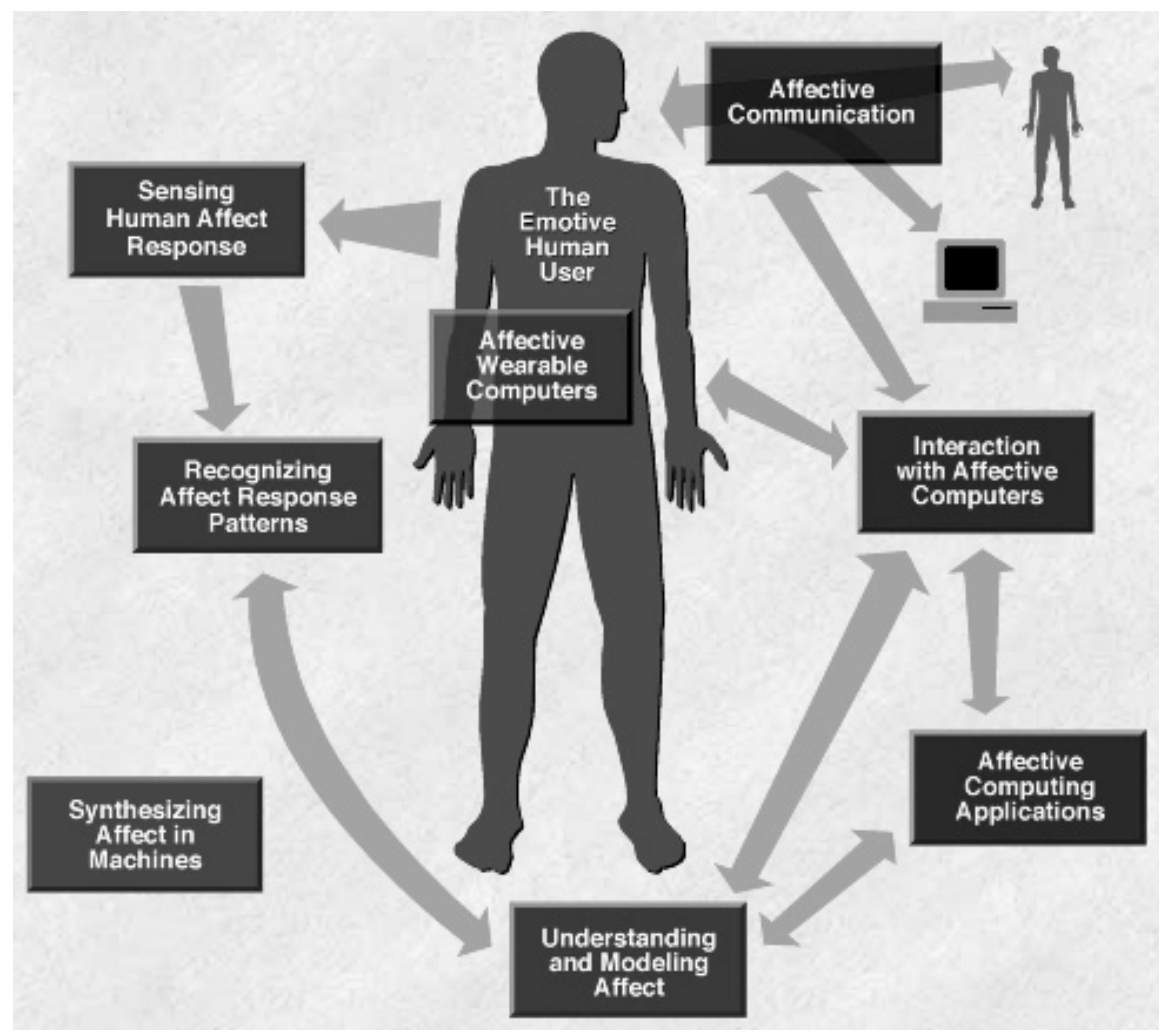

Fig. 1. The research areas of affective computing as visualized by MIT 


\subsection{Detecting and Recognizing Emotional Information}

Detecting emotional information usually involves passive sensors which capture data about the user's physical state or behaviour. The data gathered is often analogous to the cues humans use to perceive emotions in others. For example, a video camera might capture facial expressions, body posture and gestures, while a microphone might capture speech. Other sensors detect emotional cues by directly measuring physiological data, such as skin temperature and galvanic resistance.

Recognizing emotional information requires the extraction of meaningful patterns from the gathered data ([21], [23]). This is done by parsing the data through various processes such as facial expression detection, gesture recognition, speech recognition, or natural language processing.

\subsection{Emotion in Machines}

By emotion in machines, we refer to the simulation of emotions. The goal of such simulation is to enrich and facilitate interactivity between human and machine. The most common and probably most complicated application of this simulation lies in the field of conversational agents. Such a simulation is closely coupled with emotional understanding and modelling as explained below. This being said it is important to mention that less sophisticated simulation approaches often produce surprisingly engaging experiences in the area of new media. It is often the case that our aim is not to fully simulate human behaviour and emotional responses, but merely to illustrate emotion in a pseudo-intelligent way that makes sense in the specific context of interaction.

\subsection{Emotional Understanding}

Emotional understanding refers to the ability of a device to not only detect emotional or affective information, but also to store, process, build and maintain an emotional model of the user. The goal is to understand contextual information about the user and her environment, and formulate an appropriate response. This is difficult because human emotions arise from complex external and internal contexts [17].

Possible features of a system which displays emotional understanding might be adaptive behaviour, for example, avoiding interaction with a user it perceives to be angry. In the case of affect-aware applications, emotional understanding makes sense in tracking the user's emotional state and adapting environment variables according to the state recognised. Questions regarding the level of detail of the tracking performed, the theoretical grounds for the analysis of the data collected and the types of potential output that would make sense for such an interactive process, are paramount. 


\section{Emotion Descriptions and Emotion Models}

Having reviewed the areas of affective computing, it is time to start focusing on the available theories, descriptions and models that can support these goals. We start with reviewing the three big groups of emotion descriptions as identified by the members of the Humaine (Human-Machine Interaction Network on Emotion) Network of Excellence.

It is important to stress the difference that exists between emotion models and emotion descriptions. By emotion descriptions we refer to different ways of representing emotions and their underlying psychological theories whereas with the term emotional models we talk about the computational modelling of these theories in specific context.

\subsection{Categorical Representations}

Categorical representations are the simplest and most widespread, using a word to describe an emotional state. Such category sets have been proposed on different grounds, including evolutionarily basic emotion categories; most frequent everyday emotions; application-specific emotion sets; or categories describing other affective states, such as moods or interpersonal stances. (Feeltrace core vocabulary in [8]; Ortony's list of emotion words in [30] and [31]; Ekman's list of six basic emotions in [12])

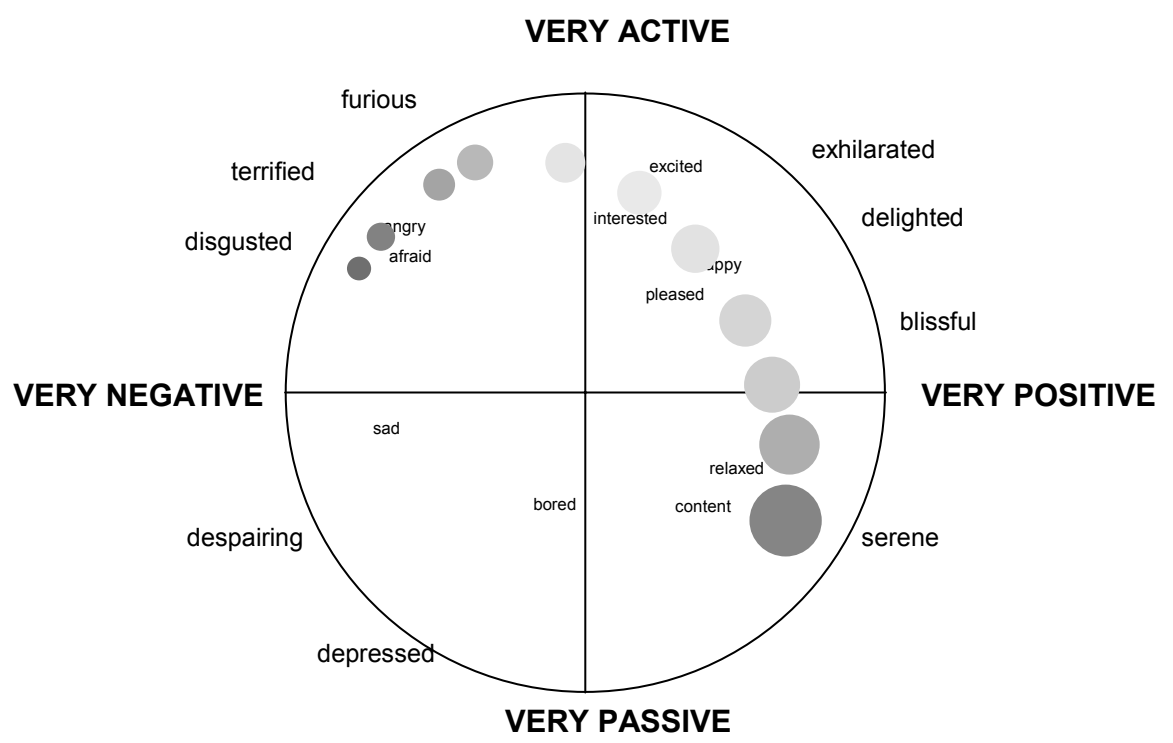

Fig. 2. A dimensional representation of emotion 


\subsection{Dimensional Descriptions}

Dimensional descriptions capture essential properties of emotional states, such as arousal (active/passive) and valence (negative/positive). Emotion dimensions can be used to describe general emotional tendencies, including low-intensity emotions.

In addition to these two, there are a number of other possible dimensions, such as power, control, or approach / avoidance, which add some refinement. The most obvious is the ability to distinguish between fear and anger, both of which involve negative valence and high activation. In anger, the subject of the emotion feels that he or she is in control; in fear, control is felt to lie elsewhere.

Dimensional representations are attractive mainly because they provide a way of describing emotional states that is more tractable than using words. This is of particular importance when dealing with naturalistic data, where a wide range of emotional states occur. Similarly, they are much more able to deal with nondiscrete emotions and variations in emotional state over time. A further attraction is the fact that dimensional descriptions can be translated into and out of verbal descriptions. This is possible because emotion words can, to an extent, be understood as referring to positions in activation-evaluation space.

\subsection{Appraisal Theories and Representations}

Appraisal theories focus on the emotion elicitation process in contrast with the previously mentioned approaches that emphasize the consequences/ symptoms of an emotional episode. Appraisal representations characterise emotional states in terms of the detailed evaluations of eliciting conditions, such as their familiarity, intrinsic pleasantness, or relevance to one's goals. Such detail can be used to characterise the cause or object of an emotion as it arises from the context, or to predict emotions in AI systems ([26],[14], [40]).

Appraisal theories are very common in emotion modelling since their structure makes it feasible for simulating their postulations in computational models. Moreover, it is often the case that an appraisal theory was formulated explicitly in order to be implemented in a computer. Such an example is the OCC theory ([30]). This is sometimes a source of confusion, since the underlying emotion theory is unavoidably very closely linked with the actual modelling approach.

According to cognitive theories of emotion ([25]), emotions are closely related to the situation that is being experienced (or, indeed, imagined) by the agent. In the following, we are going to outline four of the most prevalent theories in the field.

\subsubsection{OCC Theory}

The theory of Ortony, Clore and Collins ([30]) assumes that emotions develop as a consequence of certain cognitions and interpretations. Therefore, it exclusively concentrates on the cognitive elicitors of emotions. The authors postulate that three aspects determine these cognitions: events, agents, and objects. Emotions represent 
valenced (positive/ negative) reactions to these perceptions of the world. One can be pleased about the consequences of an event or not (pleased/displeased); one can endorse or reject the actions of an agent (approve/disapprove) or one can like or not like aspects of an object (like/dislike).

A further differentiation consists of the fact that events can have consequences for others or for oneself and that an acting agent can be another or oneself. The consequences of an event for another can be divided into desirable and undesirable; the consequences for oneself as relevant or irrelevant expectations. Relevant expectations for oneself finally can be differentiated again according to whether they actually occur or not (confirmed/disconfirmed).

With the help of such a formal system, a computer should be able to draw conclusions about emotional episodes which are presented to it. The authors are not interested in the question if machines are actually 'experiencing' emotions. Rather, they only focus on the ability to understand emotions, reason about them and express them.

\subsubsection{Scherer's Appraisal Theory}

Scherer's appraisal theory ([40], [42]) is more commonly known as the component process model. For Scherer five functionally defined subsystems are involved with emotional processes. An information-processing subsystem evaluates the stimulus through perception, memory, forecast and evaluation of available information. A supporting subsystem adjusts the internal condition through control of neuroendocrine, somatic and autonomous states. A leading subsystem plans, prepares actions and selects amongst competitive motives. An acting subsystem controls motor expression and visible behaviour. A monitor subsystem finally controls the attention assigned to the present states and passes the resulting feedback on to the other subsystems.

Scherer is especially interested in the information-processing subsystem. According to his theory, this subsystem is based on appraisals which Scherer calls stimulus evaluation checks (SEC). The result of these SECs causes again changes in the other subsystems.

Scherer enumerates five substantial SECs, four of which possess further subchecks. The novelty check decides whether external or internal stimuli have changed; its sub-checks are suddenness, confidence and predictability. The intrinsic pleasantness check specifies whether the attraction is pleasant or unpleasant and causes appropriate approximation or avoidance tendencies. The goal significance check decides whether the event supports or prevents the goals of the person; its sub-checks are goal relevance, probability of result, expectation, support character and urgency. The coping potential check determines to what extent the person believes he or she has events under control; its sub-checks are agent, motive, control, power and adaptability. The compatibility check finally compares the event with internal and external standards; its sub-checks are externality and internality. 
According to Scherer, each emotion can thus be clearly determined by a combination of the SECs and sub-checks. An appropriate table with such allocations can be found in ([41]).

\subsubsection{Roseman's Theory}

Roseman first presented his theory in the late seventies and then modified it several times in later years. Five cognitive dimensions determine whether an emotion arises and which one it is. These dimensions were re-visited several times. Nevertheless the model proposed was never empirically validated thus leading to new revisions/ additions ([34], [35]).

\subsubsection{Frijda's Theory}

Frijda $[14,15]$ points out that the word "emotion" does not refer to a "natural class" and that it is not able to refer to a well-defined class of phenomena which are clearly distinguishable from other mental and behaviour events. For him, therefore, the process of emotion emergence is of larger interest.

The centre of Frijda's theory is the term concern. A concern is the disposition of a system to prefer certain states of the environment and of its own organism over the absence of such conditions. Concerns produce goals and preferences for a system. If the system has problems in realizing these concerns, emotions develop.

The strength of such an emotion is determined essentially by the strength of the relevant concern(s). For Frijda, emotions are necessary for systems that realize multiple concerns in an uncertain environment. If a situation occurs, in which the realization of these concerns appears endangered, so-called action tendencies develop. These action tendencies are linked closely with emotional states and serve as a safety device for what Frijda calls concern realization (CR).

\section{Computational Models}

Emotion models are computational approaches that are based on these descriptions/ theories (often combinations of more than one type of description). They aspire to validate them and possibly to extend them. They allow the simulation of behaviour and aid in both recognising and understanding human emotions as well as generating synthetic emotional responses. Keeping in mind these descriptions, emotion models can be divided into two categories:

- ones that take into account the situations that initiate the emotions and how they are construed by the experiencer and focus on the predicted emotion - from now on we shall refer to them as 'deep models'.

- ones that deal with the 'results' of an emotional episode i.e. facial expression/ voice etc. - from now on we shall refer to them as 'shallow models'. 
What is the motivation for the development of a computational model implementing a particular emotion theory, or attempting to account for particular data? On a research level, it provides an opportunity for validation of the theory's claims. It also allows for the generation of alternative hypotheses explaining specific affective data or phenomena. The aim of this chapter is to investigate existing modelling approaches and to isolate the ones that would potentially meet a particular set of requirements. In order to do that we shall first go through a general overview of emotion modelling literature.

A number of computational models addressing emotion have been developed in cognitive science and AI. These models range from individual processes to integrated architectures, and explore several of the emotion theories outlined above. One thing that differentiates these modelling approaches is the level of abstraction. At the higher level of abstraction are architecture-level models that embody emotional processing. At an intermediate level of abstraction are task-level models of emotion, which focus on addressing a single task, such as natural language understanding or specific problem solving. At lower levels of abstraction are mechanism-level models, which attempt to emulate some specific aspect of affective processing. The level of abstraction is found to be a key criterion in the selection of the appropriate models for an actual application.

According to a review on emotional models by Hudlicka ([19]) the most frequently modelled process has been cognitive appraisal, whereby external and internal stimuli (emotion elicitors) are mapped onto a particular emotion. Several alternatives have been hypothesized for these processes in the psychological literature ([14], [26], [43], [30], [39]). A number of these models have been implemented, both as stand-alone versions, and integrated within larger agent architectures (e.g. [45], [6], [7], [11], [5]). The most frequently implemented theory is the OCC appraisal model ([30]), implemented in a number of systems and agents ([3], [1], [13]). Other emotion model implementations include models of emotions based on facial expression ([21] on recognition; [33] on synthesis), models of emotion based on blends of basic emotions ([33]), models of emotion based on Scherer's appraisal theory ([28]), models as goal management mechanisms ([16]), models of interaction of emotion and cognition ([2]), explicit models of the effects of emotion on cognitive processes ([20]), and effects of emotions on agent's belief generation ([17]).

Examples of integrated architectures focusing on emotion include most notably the work of Sloman and colleagues ([38]), but also more recent efforts to integrate emotion effects in Soar (a general cognitive architecture for developing systems that exhibit intelligent behaviour) by Jones and colleagues ([22]).

\section{$7 \quad$ Is There a 'Right' Model for an Application?}

As it is made clear throughout this chapter the choice of the emotion modelling approach for practical purposes does not have a one-phrase answer. In order to 
facilitate model choice, by pinpointing which emotion model is more fitting for which type of application, we propose a participatory design approach.

\subsection{Participatory Design}

Participatory design has been characterised as the 'third space' in Human Computer Interaction ([29]). In the world of software development, participatory design is an approach to design that attempts to involve the end users in the design process in a pro-active manner; this helps ensure that the outcome designed meets their needs and is usable. In participatory design, end-users (putative, potential or future) are invited to cooperate with researchers and developers during an innovation process. Potentially, they participate during several stages of an innovation process: they participate during the initial exploration and problem definition both to help define the problem and to focus ideas for solution, and during development, they help evaluate proposed solutions ([37]).

Participatory design can be seen as a way to move end-users views into the world of researchers and developers, whereas an empathic design approach would move researchers and developers into the world of end-users. Participatory design is expected to add an extra feedback loop in the showcase design phase. Each showcase designer will be able to put forward the detailed requirements that arise from corresponding use case scenarios. These use case scenarios can consequently be tested with the participation and active feedback of actual users. Such an approach is feasible thanks to their componential/modular structure. This structure allows for flexible decisions in the design process governed by the vital comments of end users. The suggested approach is similar to a formative evaluation of a system under development. It empowers users to engage in informed participation rather than being restricted to the use of existing, fully deployed and unchangeable systems.

This approach will help address open-ended and possibly multidisciplinary design problems that typically involve a combination of social and technological issues and don't have right or wrong answers.

\subsection{Model Requirements vs. Application / Showcase Requirements}

It has been made apparent throughout this text that the choice of the emotion modelling approach allows for flexible decisions and combinations of approaches in order to meet the showcase requirements. At this point we are going to identify some core requirements that make sense in all the showcases and then look into specific requirements that arise in each showcase separately.

Since we are talking about new media applications where interaction with the users is in the centre of attention we have to make sure it abides all basic usability restrictions. It is also crucial that the level of intrusiveness in the tracking process of emotion recognition is kept low. The ease of use of each showcase setup might 
conflict with the level of user engagement it manages to attain. It is obvious that the quality of the experience is prioritised highly and thus it is important to define the thresholds of acceptable intrusiveness conditions and complexity of setups in order to ensure it.

As underlined previously, context information during emotion modelling facilitates the process by providing knowledge about the event focus and the way it is appraised. Thus for each showcase the specific context is expected to dictate its own requirements towards the level of abstraction to be adopted, the deep or shallow modelling approach and the way temporal evolution of emotional measurements is dealt with. It would make sense to claim that a more detailed modelling approach is matching to the interactive-TV showcase whereas a more elementary modelling approach would correspond to a public space installation where the number of users and the environment noise constrain the level of detail of information collected.

\section{References}

[1] Andre, E., Klesen, M., Gebhard, P., Allen, S., Rist, T.: Integrating models of personality and emotions in lifelike characters. In: Paiva, A. (ed.) IWAI 1999. LNCS, vol. 1814, pp. 150-165. Springer, Heidelberg (2000)

[2] Araujo, A.F.R.: Emotions influencing cognition. In: WAUME '93, Workshop on Architectures Underlying Motivation and Emotion, The University of Birmingham, Birmingham, UK (1993)

[3] Bates, J., Loyall, A.B., Reilly, W.S.: Integrating reactivity, goals, and emotion in a broad agent. In: Proceedings of the 14th Meeting of the Cognitive Science Society, Boulder, CO (1992)

[4] Breckler, S.J.: Empirical validation of affect, behaviour and cognition as distinct attitude components. Journal of Personality and Social Psychology 47(6), 1191-1205 (1984)

[5] Breazeal, C.: Emotion and sociable humanoid robots. International Journal of HumanComputer Studies 59(1-2), 119-155 (2003)

[6] Canamero, D.: Issues in the design of emotional agents. In: Proceedings of Emotional and Intelligent: The Tangled Knot of Cognition, AAAI Fall Symposium, TR FS-9803, pp. 49-54. AAAI Press, Menlo Park (1998)

[7] Castelfranchi, C.: Affective appraisal versus cognitive evaluations in social emotions and interactions. In: Paiva, A. (ed.) Affective Interactions: Towards a New Generation of Affective Interfaces, Springer, New York (2000)

[8] Cowie, R., Douglas-Cowie, E., Apolloni, B., Taylor, J., Romano, A., Fellenz, W.: What a neural net needs to know about emotion words. In: Mastorakis, N. (ed.) Computational intelligence and applications, pp. 109-114. World Scientific Engineering Society (1999)

[9] Cowie, R., Douglas-Cowie, E., Tsapatsoulis, N., Votsis, G., Kollias, S., Fellenz, W., Taylor, J.G.: Emotion recognition in human-computer interaction. IEEE Signal Processing Magazine 18, 32-80 (2001)

[10] Damasio, A.: Looking for Spinoza: Joy, Sorrow, and the Feeling Brain. Harcourt Press, Orlando (2003) 
[11] De Rosis, F., Pelachaud, C., Poggi, I., Carofiglio, V., Carolis, B.D.: From Greta's mind to her face: modeling the dynamics of affective states in a conversational embodied agent. International Journal of Human-Computer Studies 59(1-2), 81-118 (2003)

[12] Ekman, P.: An argument for basic emotions. Cognition \& Emotion 6, 169-200 (1992)

[13] Elliott, C., Rickel, J., Lester, J.C.: Lifelike pedagogical agents and affective computing: An exploratory synthesis. In: Veloso, M.M., Wooldridge, M.J. (eds.) Artificial Intelligence Today. LNCS (LNAI), vol. 1600, pp. 195-211. Springer, Heidelberg (1999)

[14] Frijda, N.H.: The Emotions. Studies in Emotion and Social Interaction. Cambridge University Press, New York (1986)

[15] Frijda, N.H.: Emotions and Beliefs: How Feelings Influence Thoughts. Cambridge University Press, Cambridge (2000)

[16] Frijda, N.H., Swagerman, J.: Can computers feel? Theory and design of an emotional system. Cognition and Emotion 1(3), 235-257 (1987)

[17] Gratch, J., Marsella, S.: A domain-independent framework for modeling emotion. Cognitive Systems Research 5, 269-306 (2004)

[18] Goldie, P.: On Personality. Rutledge, New York (2004)

[19] Hudlicka, E.: To feel or not to feel: The role of affect in human-computer interaction. International Journal of Human-Computer Studies 59, 1-32 (2003)

[20] Hudlicka, E.: Increasing SIA architecture realism by modeling and adapting to affect and personality. In: Dautenhahn, K., Bond, A.H., Canamero, L., Edmonds, B. (eds.) Multiagent Systems, Artificial Societies, and Simulated Organizations, Kluwer Academic Publishers, Dordrecht (2002)

[21] Ioannou, S., Raouzaiou, A., Tzouvaras, V., Mailis, T., Karpouzis, K., Kollias, S.: Emotion recognition through facial expression analysis based on a neurofuzzy network. Neural Networks (Special Issue on Emotion: Understanding \& Recognition) 18(4), 423-435 (2005)

[22] Jones, R., Henninger, A., Chown, E.: Interfacing emotional behavior moderators with intelligent synthetic forces. In: Proceedings of the 11th Conference on Computer Generated Forces and Behaviour Representation, Orlando, FL (2002)

[23] Karpouzis, K., Caridakis, G., Kessous, L., Amir, N., Raouzaiou, A., Malatesta, L., Kollias, S.: Modeling naturalistic affective states via facial, vocal, and bodily expressions recognition. In: Huang, T.S., Nijholt, A., Pantic, M., Pentland, A. (eds.) ICMI/IJCAI Workshops 2007. LNCS (LNAI), vol. 4451, pp. 91-112. Springer, Heidelberg (2007)

[24] Kant, I.: Critique of Judgment (1790) (Trans. Werner S. Pluhar). Hackett Publishing, Indianapolis (1987)

[25] Lazarus, R.S., Folkman, S.: Transactional theory and research on emotions and coping. European Journal of Personality 1, 141-169 (1987)

[26] Lazarus, R.S.: Emotion and Adaptation. Oxford University Press, New York (1991)

[27] Leventhal, H., Scherer, K.R.: The relationship of emotion to cognition: a functional approach to a semantic controversy. Cognition and Emotion 1, 3-28 (1987)

[28] Malatesta, L., Raouzaiou, A., Karpouzis, K., Kollias, S.: MPEG-4 facial expression synthesis. Personal and Ubiquitous Computing (Special issue on Emerging Multimodal Interfaces) 13(1), 77-83 (2007)

[29] Muller, M.J.: Participatory Design: The Third Space in HCI - Handbook of HCI. Erlbaum, Mahwah (2003) 
[30] Ortony, A., Collins, A., Clore, G.L.: The Cognitive Structure of Emotions. Cambridge University Press, Cambridge (1988)

[31] Ortony, A., Turner, T.J.: What's basic about basic emotions? Psychological Review 97, 315-331 (1990)

[32] Picard, R.W.: Affective Computing. MIT Press, Cambridge (1997)

[33] Raouzaiou, A., Tsapatsoulis, N., Karpouzis, K., Kollias, S.: Parameterized facial expression synthesis based on MPEG-4. EURASIP Journal on Applied Signal Processing 2002(10), 1021-1038 (2002)

[34] Roseman, I.J.: The structure of emotion antecedents: Individual and cross-cultural differences. New approaches to emotion structure and process. Symposium conducted at the 92nd Annual Convention, American Psychological Association, Toronto, Canada (1984)

[35] Roseman, I.J.: The emotion system: Strategies for coping with crises and opportunities. Paper presented at Department of Psychology, The Graduate Center, City University of New York (1990)

[36] Rusting, C.: Personality, mood, and cognitive processing of Emotional information: three conceptual frameworks. Psychological Bulletin 124, 165-196 (1998)

[37] Schuler, D., Namioka, A. (eds.): Participatory Design: Principles and Practices. Lawrence Erlbaum Associates, Hillsdale (1993)

[38] Sloman, A.: Beyond shallow models of emotions. Cognitive Processing 2(1), 177-198 (2001)

[39] Smith, C.A., Kirby, L.D.: Affect and appraisal. In: Forgas, J.P. (ed.) Feeling and Thinking: The Role of Affect in Social Cognition, Cambridge University Press, Cambridge (2000)

[40] Scherer, K.R.: Toward a dynamic theory of emotion: The component process model of affective states. Geneva Studies in Emotion and Communication 1, 1-98 (1987)

[41] Scherer, K.R.: Criteria for emotion-antecedent appraisal: A review. In: Hamilton, V., Bower, G.H., Frijda, N.H. (eds.) Cognitive perspectives on emotion and motivation, pp. 89-126. Kluwer Academic Publishers, Dordrecht (1988)

[42] Scherer, K.R.: Appraisal considered as a process of multi-level sequential checking. In: Scherer, K.R., Schorr, A., Johnstone, T. (eds.) Appraisal processes in emotion: Theory, methods, research, pp. 92-120. Oxford University Press, New York (2001)

[43] Scherer, K.R.: Feelings integrate the central representation of appraisal-driven response organization in emotion. In: Manstead, A.S.R., Frijda, N.H., Fischer, A.H. (eds.) Feelings and emotions The Amsterdam symposium, pp. 136-157. Cambridge University Press, Cambridge (2004)

[44] Scherer, K.R.: Unconscious processes in emotion: The bulk of the iceberg. In: Niedenthal, P., Feldman-Barrett, L., Winkielman, P. (eds.) The unconscious in emotion, Guilford, New York (2005)

[45] Velasquez, J.: Modeling Emotions and Other Motivations in Synthetic Agents. In: Proceedings of AAAI-97, Providence, RI, pp. 10-15 (1997) 